\title{
Atypical Chikungunya virus infections: clinical manifestations, mortality and risk factors for severe disease during the 2005-2006 outbreak on Réunion
}

\section{A. ECONOMOPOULOU ${ }^{1,2 *}$, M. DOMINGUEZ ${ }^{1,3}$, B. HELYNCK ${ }^{1}$, D. SISSOKO ${ }^{4}$, O. WICHMANN ${ }^{5}$, P. QUENEL ${ }^{6}$, P. GERMONNEAU ${ }^{1}$ AND I. QUATRESOUS ${ }^{1}$}

${ }^{1}$ Institute de Veille Sanitaire, France

${ }^{2}$ European Programme for Intervention Epidemiology Training (EPIET), European Centre for Disease

Prevention and Control, Stockholm, Sweden

${ }^{3}$ Training Programme in Field Epidemiology, Institut de Veille Sanitaire, France

${ }^{4}$ Cellule Interrégionale d'Epidémiologie Réunion-Mayotte

${ }^{5}$ Department for Infectious Disease Epidemiology, Robert Koch Institute, Berlin, Germany

${ }^{6}$ Cellule Interrégionale d'Epidémiologie Antilles-Guyane

(Accepted 9 July 2008; first published online 11 August 2008)

\section{SUMMAR Y}

In April 2005, an outbreak of Chikungunya fever occurred on the island of Réunion in the Indian Ocean. During winter 2005, six patients developed meningoencephalitis and acute hepatitis due to Chikungunya virus. Our objectives were to determine the incidence and mortality of atypical Chikungunya viral infections and to identify risk factors for severe disease. A hospital-based surveillance system was established to collect data on atypical Chikungunya cases. Case reports, medical records and laboratory results were reviewed and analysed. We defined an atypical case as one in which a patient with laboratory-confirmed Chikungunya virus infection developed symptoms other than fever and arthralgia. We defined a severe atypical case as one which required maintenance of at least one vital function. We recorded 610 atypical cases of Chikungunya fever: 222 were severe cases, 65 affected patients died. Five hundred and forty-six cases had underlying medical conditions (of which 226 suffered from cardiovascular, 147 from neurological and 150 from respiratory disorders). Clinical features that had never been associated with Chikungunya fever were recorded, such as bullous dermatosis, pneumonia, and diabetes mellitus. Hypertension, and underlying respiratory or cardiological conditions were independent risk factors for disease severity. The overall mortality rate was $10 \cdot 6 \%$ and it increased with age. This is the first time that severe cases and deaths due to Chikungunya fever have been documented. The information presented in this article may assist clinicians in identifying the disease, selecting the treatment strategy, and anticipating the course of illness.

Key words: Chikungunya virus, clinical manifestations, outbreak, Réunion, severity.

\section{INTRODUCTION}

Chikungunya fever is an arboviral disease transmitted to humans by infected mosquitoes of the Aedes genus

* Author for correspondence: Dr A. Economopoulou, Hellenic Center for Disease Control and Prevention, 9 Polytecneiou St, 10433, Athens, Greece.

(Email: a_economopoulou@yahoo.gr)
[1]. Since the first isolated incident in 1952, several outbreaks of the disease, caused by this Alphavirus, have been documented throughout South-East Asia, and Africa [2-5]. After an incubation period of 1-12 days, patients usually develop a sudden onset of fever, headache and arthralgia [6]. Conjunctivitis and rash are also common features of Chikungunya fever. The 
disease is not generally considered life-threatening, and prior to 2005 severe clinical forms of the infection were rarely described [7].

In April 2005 an outbreak of Chikungunya fever first manifested itself on the French island territory of Réunion, located in the Archipelago of Mascareignes (South-West Indian Ocean) [7]. According to reports from the Réunion-Mayotte Inter-Regional Epidemiological Unit (IREU), as of mid-April 2006, $266000 /$ $785221(34 \%)$ inhabitants had been infected by the virus [8]. Health-care facilities on Réunion are similar to those of mainland France. The island is divided into 24 counties grouped in four regions, each region having a hospital.

In autumn 2005 during the early warm season six patients who were hospitalized with Chikungunya fever on Réunion developed atypical clinical features, four of them developed meningoencephalitis and two acute hepatitis. In response to this observation, we established a hospital-based surveillance system to identify the hospitalized patients who developed clinical features of Chikungunya fever other than the typical fever-arthralgia. The objectives of the surveillance system were to describe the atypical clinical forms of Chikungunya fever, to determine its incidence and mortality and to assess risk factors for severe disease and death.

\section{MATERIALS AND METHODS}

Between 1 February and 31 May 2006, epidemiologists from the Réunion-Mayotte IREU, on a weekly basis, contacted referring physicians from all four hospitals on the island inquiring about newly hospitalized Chikungunya fever cases with atypical disease symptoms. In addition, a retrospective data collection was conducted to identify atypical cases that occurred between 1 April 2005 and 31 March 2006.

Chikungunya fever cases required biological confirmation either by serological testing (IgM-antibody titres indicating acute or recent infection), or by detecting the presence of Chikungunya virus genome in any body specimen using real-time reverse transcription-polymerase chain reaction assays (RT-PCR). Samples of blood serum, cerebrospinal fluid or serum from bullous lesions were tested with an IgM-capture enzyme-linked immunosorbent assay (ELISA) for antibodies against Chikungunya virus and by a standard RT-PCR technique [9]. A case was considered to be biologically confirmed if any one of the above performed tests in any clinical specimen were positive.

We defined as an 'atypical case' of Chikungunya fever as one in which a patient hospitalized from April 2005 onwards on Réunion, developed, apart from fever and arthralgia, one or more other symptoms and had laboratory evidence of acute or recent Chikungunya virus infection. This atypical case was defined as a 'severe case' of Chikungunya fever requiring the maintenance of at least one vital function. All patients with Chikungunya infection that died during the course of the disease were defined as severe cases.

Case reports, medical records, and the results of biological tests were reviewed for each Chikungunya fever case by an epidemiologist in order to identify patients with atypical manifestations. For each atypical case, a standard questionnaire was completed. Signs or symptoms not mentioned in medical records were considered as absent. In order to confirm the findings extracted from the medical records the treating neurologist, cardiologist, and intensive care physician of the reanimation ward of the two main hospitals were also contacted.

Data on underlying conditions such as hypertension, diabetes, cardiovascular and respiratory disorders, cancer and alcoholism were recorded from the patient's medical records. Patients were classified as having meningoencephalitis, if the cerebrospinal fluid test was positive by PCR or IgM-capture ELISA. A patient with diabetes mellitus and hypertension who also presented neurological features was not classified as having a neurological disorder owing to the infection. A patient with a simultaneous infection was characterized as having a concomitant disease. Only adult patients (i.e. aged $>15$ years) have been included in the present analysis. Clinical manifestation in children will be reported elsewhere.

Analysis of the collected data was performed using Stata statistical software, version 8.0 (StataCorp LP, College Station, TX, USA). Denominators that were used to calculate proportions for the reported data varied, based on available information. Incidence and mortality were calculated according to the 2001 Réunion population census data [8]. A multivariate analysis was conducted to examine the association between age, sex, underlying health status, alcoholism, diabetes, use of non-steroidal anti-inflammatory drugs (NSAIDs) and disease severity and death in all notified atypical cases. For this purpose, logistic regression models were developed with stepwise 


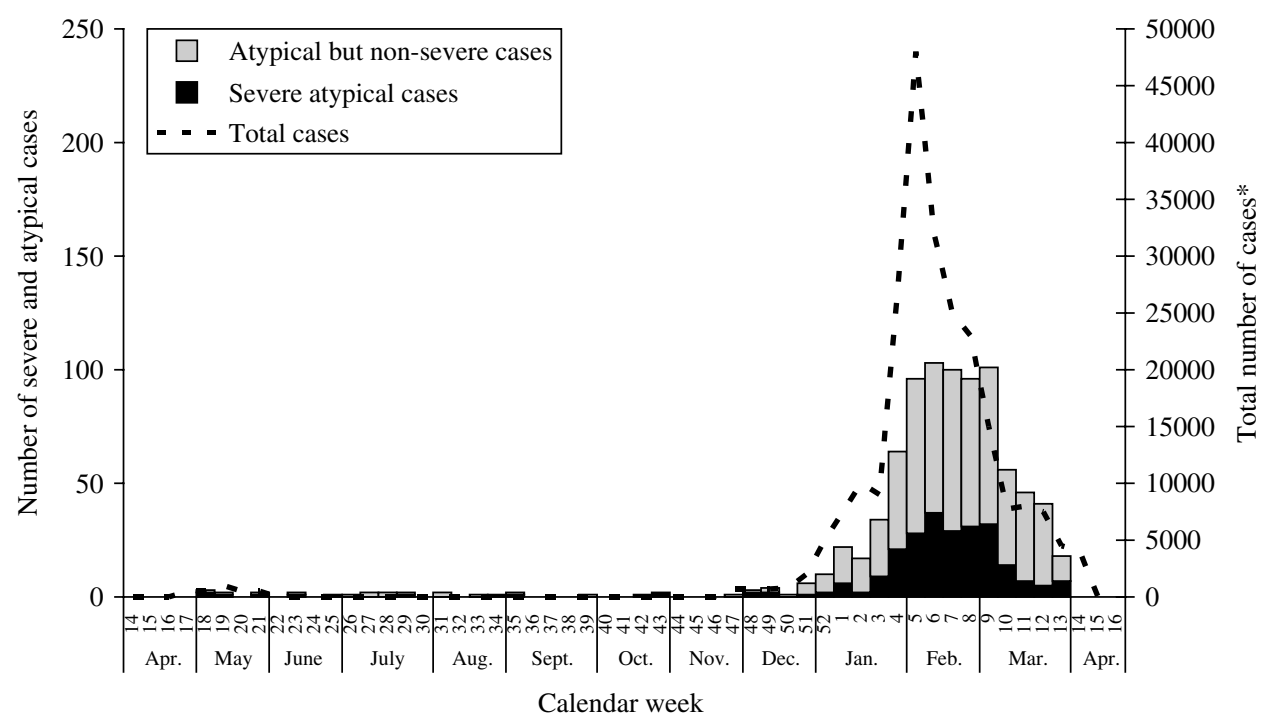

Fig. 1. Number of atypical and severe Chikungunya cases $(n=610)$ and total number of notified Chikungunya cases by calendar week, Réunion, April 2005 to April 2006. * Data from total weekly notified cases have been adapted from Martin et al. [9].

backwards removal of non-significant variables. Original models included gender, age and all variables with a $P$ value $<0.2$ in the bivariate analysis. A $P$ value $<0.05$ was considered statistically significant.

\section{RESULTS}

Between 1 April 2005 and 31 March 2006, a total of 878 patients with atypical Chikungunya symptoms were investigated. Over the same period, the incidence of atypical cases on Réunion was 112/100000 inhabitants. Taking into consideration the estimates for the total number of infected people reported previously, the proportion of atypical cases in all Chikungunya patients was $0 \cdot 3 \%$ [8]. The majority of hospitalized atypical cases occurred in February and March 2006; this time period coincides with the period of the highest reporting of Chikungunya fever cases on Réunion (Fig. 1) [9, 10].

In all atypical cases, 610 were adults, 224 were children aged $<16$ years, and 44 were newborns aged $<10$ days who were infected by mother-to-child transmission. The present study presents the analysis of exclusively adult patient data.

The median age of the cases was 70 years (range 15-95) and the sex ratio $(M / F)$ was 0.8 . In the 610 atypical cases, $222(36 \%)$ were severe cases. Eighty-four (14\%) were admitted to an intensive care unit and 65 of the severe cases ( $29 \%$ ) died, accounting for an overall case-fatality rate of $10 \cdot 7 \%$.
The incidence of atypical cases, severe cases, and the mortality rate increased with age. Therefore, the incidence of the disease was more than 30 times higher in patients aged $\geqslant 65$ years compared to those aged $<45$ years (Table 1 ).

\section{Clinical features and underlying health status}

Of the 610 atypical cases, $546(89 \%)$ had underlying medical conditions, $479(78 \%)$ were on medication prior to hospitalization, $84(14 \%)$ used NSAIDs and $88(14 \%)$ were alcohol abusers. The recorded underlying medical conditions are summarized in Table 2 .

Almost all patients during physical examination on admission presented fever $(90 \%)$. Other recorded symptoms included malaise $(45 \%)$, altered mental status $(24 \%)$, diarrhoea or vomiting (18\%), dyspnoea $(15 \%)$, myalgia $(13 \%)$, chest pain $(4 \%)$, gastrointestinal bleeding $(4 \%)$, and haematuria $(1 \%)$.

Atypical clinical features recorded during hospitalization are summarized in Table 3. In 120 patients hospitalized with pre-renal acute renal failure, 41 $(34 \%)$ presented an exacerbation of a pre-existing renal disorder. Of these 36 had chronic renal failure, two had a nephropathy and three had a history of renal transplantation. In 102 patients hospitalized with pneumonia, 17 had a history of chronic obstructive pulmonary disorder (COPD) and three had asthma. 
Table 1. Age group-specific frequency and incidence (per 100000 inhabitants) of notified atypical Chikungunya virus infections in adults, Réunion, 2005-2006

\begin{tabular}{|c|c|c|c|c|c|}
\hline \multirow[b]{2}{*}{$\begin{array}{l}\text { Age } \\
\text { group (yr) }\end{array}$} & \multirow[b]{2}{*}{$\begin{array}{l}\text { Population } \\
\text { of Réunion }\end{array}$} & \multicolumn{4}{|c|}{ Number (incidence/100 000 inhabitants) of reported atypical cases } \\
\hline & & $\begin{array}{l}\text { All atypical } \\
\text { cases }\end{array}$ & $\begin{array}{l}\text { Severe atypical } \\
\text { cases }\end{array}$ & $\begin{array}{l}\text { Cases requiring } \\
\text { intensive care }\end{array}$ & Deaths \\
\hline $15-24$ & 136781 & $21(15)$ & $6(4)$ & $3(2)$ & $1(0 \cdot 7)$ \\
\hline $25-44$ & 232657 & $46(20)$ & $14(6)$ & $8(3)$ & $4(2)$ \\
\hline $45-64$ & 149291 & 173 (116) & $57(38)$ & $38(26)$ & $19(13)$ \\
\hline$\geqslant 65$ & 58851 & $370(629)$ & $145(246)$ & $35(60)$ & $41(69)$ \\
\hline Total & 577580 & $610(106)$ & $222(38)$ & $84(15)$ & $65(11)$ \\
\hline
\end{tabular}

Table 2. Underlying medical conditions of 610 atypical adult Chikungunya patients (multiple entries possible)

\begin{tabular}{ll}
\hline \hline Underlying medical conditions & Number $(\%)$ \\
\hline Hypertension & $329(54)$ \\
Diabetes mellitus & $242(39)$ \\
Cardiovascular diseases & $200(32)$ \\
Neurological disorders & $142(23)$ \\
Chronic pulmonary diseases & $99(16)$ \\
Alcohol abuse & $88(14)$ \\
Kidney diseases & $76(12)$ \\
Cancer & $22(4)$ \\
Allergy & $22(4)$ \\
Concomitant diseases & $22(4)$ \\
Total & $546(89)$ \\
\hline \hline
\end{tabular}

A rash which was described as an erythematous macular, papular or morbiliform eruption was reported in $104(17 \%)$ patients and affected one or more of the following areas: neck, trunk, arms and legs. Four per cent of the cases presented itching and desquamation. Seventeen cases presented bullous dermatosis, of which $3 \%$ required intensive care unit admission. For one case a hyperpigmentation of the nose was described.

A total of 131 of the atypical cases presented glycaemic imbalance, $27(20 \%)$ of them were diagnosed with diabetes mellitus for the first time. Seventy-three of the atypical cases were observed to have a greater than threefold increase of liver enzymes. However, information on liver enzymes was only available for $64 \%$ of the cases.

Of the 226 patients with atypical Chikungunya fever presenting cardiovascular disease, $110(49 \%)$ had an underlying cardiac condition, 137 (61\%) had hypertension, and 66 of these $(48 \%)$ also had diabetes mellitus. Of the 84 cases with heart failure, $29(35 \%)$ had an underlying cardiomyopathy, 10 (12\%) coronary artery disease, six (7\%) valvular disease, four $(5 \%)$ a history of myocardial infarction and one of arrhythmias. Of the 44 cases with arrhythmias, seven had arrhythmias reported as a medical underlying condition, six cardiopathy, two valvulopathy, and one heart failure. Of the 35 cases with myocarditis or pericarditis, seven had a underlying cardiopathy, two arrhythmias, and two had a history of myocardial infarction. Of the 25 cases diagnosed with angina pectoris, $15(60 \%)$ had a history of coronary artery disease. Four cases presented acute myocardial infarction; of which two had a history of coronary artery disease.

Of 147 cases with neurological disorders, 25 (17\%) had underlying neurological conditions, while 71 $(48 \%)$ also had a diagnosis of hypertension, and 36 (24\%) of diabetes mellitus. Of the 69 cases with encephalitis, $12(17 \%)$ had underlying neurological conditions: five $(7 \%)$ had suffered a stroke and five (7\%) had a history of epilepsy. Of these $34(49 \%)$ had a diagnosis of hypertension. Of the 15 cases with meningoencephalitis, one had history of cysticercosis, one of hydrocephalus, one of myasthenia gravis and eight a diagnosis of hypertension. Eight $(8 \%)$ out of 12 cases who developed seizures were previously healthy.

\section{Disease severity and prognosis}

The univariate analysis revealed that atypical cases aged 40-60 years were 2.5 times more likely to develop severe disease than those aged $<40$ years $[95 \%$ confidence interval (CI), 1·13-5.48). Atypical cases aged $\geqslant 60$ years were 1.6 times more likely to develop severe disease than those aged $<40$ years $(95 \%$ 
Table 3. Clinical features observed in 610 atypical Chikungunya cases

\begin{tabular}{|c|c|}
\hline Clinical feature & $\begin{array}{l}\text { Number of } \\
\text { observations }(\%)\end{array}$ \\
\hline Cardiovascular disorders & $226(37)$ \\
\hline Heart failure & $84(13)$ \\
\hline Arrhythmias & $44(7)$ \\
\hline Myocarditis/pericarditis & $35(6)$ \\
\hline Blood pressure instability & $34(6)$ \\
\hline Coronary artery disease & $25(4)$ \\
\hline Acute myocardial infarction & $4(1)$ \\
\hline Neurological disorders & $147(24)$ \\
\hline Encephalitis & $69(11)$ \\
\hline Malaise & $25(4)$ \\
\hline Meningoencephalitis & $15(2)$ \\
\hline Epileptic seizures & $12(2)$ \\
\hline Syndrome of meningeal irritation & $8(1)$ \\
\hline Hyperaesthesia & $8(1)$ \\
\hline Guillain-Barré syndrome & $4(1)$ \\
\hline Cerebellar syndrome & 3 \\
\hline Stroke & 2 \\
\hline Myelomeningoencephalitis & 1 \\
\hline Pre-renal failure & $120(20)$ \\
\hline Pneumonia & $102(17)$ \\
\hline Skin affections & $104(17)$ \\
\hline Respiratory failure & $48(8)$ \\
\hline $\begin{array}{l}\text { Exacerbation of chronic renal } \\
\text { failure }\end{array}$ & $41(7)$ \\
\hline Hepatic insufficiency & $22(4)$ \\
\hline Bullous dermatosis & $17(3)$ \\
\hline Toxic hepatitis & $16(3)$ \\
\hline Pancreatitis & $12(2)$ \\
\hline $\begin{array}{l}\text { Syndrome of inappropriate } \\
\text { antidiuretic hormone secretion }\end{array}$ & $6(1)$ \\
\hline Hypoadrenalism & $6(1)$ \\
\hline Subacute hepatitis & $5(1)$ \\
\hline
\end{tabular}

Categories shown are not mutually exclusive, since some cases presented more than one features.

CI 1.08-2.27). In multivariate analysis, underlying respiratory diseases, the use of NSAIDs prior to hospitalization, hypertension and underlying cardiac disorders were independently associated with disease severity (Table 4).

Five out of $65(8 \%)$ reported death incidents occurred in people who were healthy prior to hospitalization. Of the deceased, two developed multiple organ failure syndrome, one meningoencephalitis, one fulminant hepatitis, and one heart failure. Causes of death are summarized in Table 5 .

Age $\geqslant 85$ years [relative risk (RR) $3 \cdot 49,95 \%$ CI $1 \cdot 5-7 \cdot 8]$ as well as alcohol abuse (RR $11 \cdot 38,95 \%$ CI $4 \cdot 88-26 \cdot 53)$ were associated with increased mortality.

\section{DISCUSSION}

Chikungunya fever is characterized as a mild febrile disease [1]. The main clinical symptoms mentioned by Robinson et al. in his original report were fever, incapacitating arthralgia, myalgia, headache, and diffuse maculopapular rash [2]. Other clinical symptoms such as diarrhoea, vomiting, persistent arthralgia, and on rare occasions bleeding, have also been described, during outbreaks or in cohorts of travellers $[6,11-13]$. Only a few case reports have been published suggesting that Chikungunya fever can be exceptionally associated with hepatitis, meningitis, encephalitis [14, 15], myocarditis and arrhythmias $[16,17]$.

This report describes the conclusions of an enhanced hospital-based surveillance system for atypical Chikungunya fever, implemented during the large Chikungunya fever outbreak on Réunion during 2005-2006. Atypical cases $(n=610)$ of Chikungunya fever were reported, 222 were severe cases, of which 65 died. Of the 610 atypical cases, $546(89 \%)$ had underlying medical conditions, 84 had taken NSAIDs and $88(14 \%)$ were alcohol abusers. In addition, the disease was associated with the presence of several underlying medical conditions, with $90 \%$ of the affected cases suffering from underlying diseases. Suprisingly, cardiovascular disorders such as arrhythmias, myocarditis, pericarditis, myocardial infarction and neurological disorders including encephalitis and meningoencephalitis, occurred in previously healthy cases. Respiratory or cardiovascular underlying medical conditions and hypertension were associated with increased risk of developing severe illness. On the contrary, diabetes mellitus, despite being commonly reported as an underlying condition, was not found to increase the risk of severe disease or death. Our study also reveals that the risk of severe Chikungunya fever increased substantially in patients who used NSAIDs prior to hospitalization. This might reflect either a more severe form of the disease or a late admission to hospital. The role of specific NSAIDs (aspirin or acetaminophen) could not be determined, as this information had not been recorded in the medical records.

This study identified a substantial number of severe manifestations and deaths due to Chikungunya fever. The reasons for these severe manifestations are unclear. It is possible that the large number of severe cases observed simply reflect the immense size of the outbreak. Alternatively, the fact that the outbreak 
Table 4. Relative risks of severity and death associated with underlying conditions and risk factors in 610 patients with atypical Chikungunya virus infection (results of multiple regression analysis)

\begin{tabular}{|c|c|c|c|c|}
\hline & \multicolumn{2}{|c|}{ Proportion with risk factor present } & \multirow[b]{2}{*}{ RR } & \multirow[b]{2}{*}{$95 \% \mathrm{CI}$} \\
\hline & $\begin{array}{l}\text { In patients } \\
\text { with the } \\
\text { outcome }\end{array}$ & $\begin{array}{l}\text { In patients } \\
\text { without the } \\
\text { outcome }\end{array}$ & & \\
\hline \multicolumn{5}{|c|}{ Outcome: severe disease $(n=222)$} \\
\hline $\begin{array}{l}\text { Underlying respiratory } \\
\text { disease }\end{array}$ & 27 & 10 & $3 \cdot 1$ & $2 \cdot 0-4 \cdot 9$ \\
\hline $\begin{array}{l}\text { Use of NSAIDs prior } \\
\text { hospitalization }\end{array}$ & 17 & 12 & $1 \cdot 8$ & $1 \cdot 1-3 \cdot 0$ \\
\hline Hypertension & 63 & 49 & $1 \cdot 7$ & $1 \cdot 2-2 \cdot 4$ \\
\hline $\begin{array}{l}\text { Underlying cardiac } \\
\text { disease }\end{array}$ & 43 & 29 & $1 \cdot 6$ & $1 \cdot 1-2 \cdot 3$ \\
\hline \multicolumn{5}{|l|}{ Outcome: death $(n=65)$} \\
\hline Alcohol abuse & 37 & 6 & $1 \cdot 4$ & $4 \cdot 9-26 \cdot 5$ \\
\hline Age $>85 \mathrm{yr}$ & 22 & 12 & $3 \cdot 5$ & $1 \cdot 6-7 \cdot 8$ \\
\hline
\end{tabular}

NSAIDs, Non-steroidal anti-inflammatory drugs; RR, relative risk; CI, confidence interval.

Table 5. Causes of deaths recorded in 65 patient with severe atypical Chikungunya fever

\begin{tabular}{ll}
\hline \hline Causes of death & Number (\%) \\
\hline Heart failure & $15(23)$ \\
Multiple organ failure syndrome & $11(18)$ \\
Toxic hepatitis & $7(11)$ \\
Encephalitis or meningoencephalitis & $6(9)$ \\
Bullous dermatosis & $6(9)$ \\
Myocarditis/pericarditis & $5(8)$ \\
Respiratory failure & $5(8)$ \\
Renal failure & $3(5)$ \\
Pneumonia & $2(3)$ \\
Acute myocardial infarction & $2(3)$ \\
Cerebrovascular disease & 1 \\
Hypothyroidism & 1 \\
Septicaemia & 1 \\
\hline \hline
\end{tabular}

occurred on an island with European health standards may have increased the chance of identifying and documenting such events [1]. On the other hand, the spread of Chikungunya virus during the outbreak on Réunion was found to have novel molecular features, which may have led to its increased virulence $[18,19]$.

During the 2005 outbreak, the incidence of atypical infections on Réunion was $0 \cdot 3 \%$ [8]. Although the overall proportion of atypical cases was low, the mortality in these cases was surprisingly high. Mortality attributed to Chikungunya fever has never been previously reported [1]. A study on crude death rates on Réunion during the outbreak supports our findings by showing 260 excess deaths between January and April 2006 [20]. Most of the deaths in our study occurred in cases with underlying medical conditions, but five deaths occurred in previously healthy cases. Mortality rate was clearly increasing with age. In atypical cases, very old age was also an independent risk factor for increased mortality rate. However, deaths were reported even in young or middle-aged persons. Eighty-four of the 222 severe cases were admitted to an intensive care unit. However, a large number of severe cases were only treated in standard wards, due to the limited admission capacity of intensive care units during the epidemic peak.

A limitation of the present study was that we only collected data from atypical Chikungunya patients through our surveillance system. For this reason, it was not possible to look for risk factors of atypical disease. The dramatically increasing incidence of agespecific atypical disease could reflect the higher occurence of underlying diseases in older ages, but could also be the result of the direct virulence of the virus. Borgherini et al. demonstrated the factors associated with hospitalization for Chikungunya fever: older age, diabetes and underlying heart disease [6]. Since 
we used a hospital-based surveillance system, atypical but mild Chikungunya fever infection might not have been detected, suggesting that the incidence of atypical infection may have been underestimated in the present study.

During this outbreak, new clinical forms of Chikungunya fever were identified such as bullous dermatosis, pneumonia, and diabetes mellitus. To our knowledge, these Chikungunya fever-associated features have never been previously reported. The resulting disease varied from mild (with fever, gastrointestinal disorders, maculopapular rush) to severe (Guillain-Barré, meningoencephalitis, heart failure, pneumonia). The underlying physiopathology for some of these disorders remains unclear. However, clinical features of atypical cases could be grouped mainly into three major categories: exacerbation of underlying medical conditions, deterioration of a previously unrecognized disorder, or exaggerated direct response to viral infection. The information presented in this paper may assist clinicians in identifying the disease, selecting the treatment strategy, and anticipating the course of illness. Today's frequent international travel from one continent to another may facilitate the introduction (spread) of the virus in areas where the vector is present. This has been recently experienced in Italy, where a traveller who returned from India was identified as the most likely source of an outbreak of Chikungunya fever which affected $>200$ people [21]. Clinicians in developed countries should be aware of this possibility and should consider the risk of a more severe disease in the elderly.

The existence of a developed medico-technical background on Réunion, the vigilant epidemiological investigation and the systematic performance of serological testing of suspected Chikungunya patients enhanced our knowledge of the unusual clinical characteristics of Chikungunya fever and gave us a better understanding of the epidemic which has led to appropriate prevention policies. The knowledge of the severity of the disease may facilitate its future management and strengthen the strategy of mitigation.

\section{ACKNOWLEDGEMENTS}

We are indebted to our colleagues at the Centre Hospitalier Départemental Félix Guyon, the Groupe Hospitalier Sud-Réunion, the Centre Hospitalier Gabriel Martin, the Centre Hospitalier
Intercommunal St André-St Benoit, particularly B. A. Gauzere, P. Poubeau, F. Binois, G. Defauw, M. Bohrer, E. Chirpaz, M. C. Jaffar, D. Ramful, J. C. Saly, O. Fels, A. Michault, J. F. Rouanet, P. Simon, A. Jacques, F. Simmonet and M. Weber for their help in data collection, and the local and public health officers for their support. Thanks are also due to A. F. Gay, D. Ilef, M. Ledrans, C. Renaudat, E. Couturier, and H. de Valk for scientific advice throughout the realization of the study.

\section{DECLARATION OF INTEREST}

None.

\section{REFERENCES}

1. Pialoux G, et al. Chikungunya, an epidemic arbovirosis. Lancet Infect Diseases 2007; 7: 319-327.

2. Robinson MC. An epidemic of virus disease in Southern Province, Tanganyika Territory, in 1952-53. I. Clinical features. Transactions of the Royal Society of Tropical Medicine and Hygiene 1955; 49: 28-32.

3. Sergon K, et al. Seroprevalence of Chikungunya virus (CHIKV) infection on Lamu Island, Kenya, October 2004. American Journal of Tropical Medicine and Hygiene 2008; 78: 333-337.

4. AbuBakar S, et al. Reemergence of endemic chikungunya, Malaysia. Emerging Infectious Diseases 2007; 13: $147-149$.

5. Halstead SB, et al. Dengue chikungunya virus infection in man in Thailand, 1962-1964. 3. Clinical, epidemiologic, and virologic observations on disease in nonindigenous white persons. American Journal of Tropical Medicine and Hygiene 1969; 18: 984-996.

6. Borgherini G, et al. Outbreak of chikungunya on Reunion Island: early clinical and laboratory features in 157 adult patients. Clinical Infectious Diseases 2007; 44: 1401-1407.

7. Charrel RN, de Lamballerie $X$, Raoult D. Chikungunya outbreaks - the globalization of vectorborne disease. New England Journal of Medicine 2007; 356: 769-771.

8. Pierre V, et al. Chikungunya epidemic on Reunion Island, 2005-2006. Presse Medicale 2006; 35: 11881189.

9. Martin DA, et al. Standardization of immunoglobulin M capture enzyme-linked immunosorbent assays for routine diagnosis of arboviral infections. Journal of Clinical Microbiology 2000; 38: 1823-1826.

10. Cordel H, et al. Imported cases of chikungunya in metropolitan France, April 2005-February 2006. Eurosurveillance 2006; 11: E060420.3 (http://www. eurosurveillance.org/ew/2006/060420.asp\#3).

11. Taubitz W, et al. Chikungunya fever in travellers: Clinical presentations and course. Clinical Infectious Diseases 2007; 45 : e1-4. 
12. Kennedy AC, Fleming J, Solomon L. Chikungunya viral arthropathy: a clinical description. Journal of Rheumatology 1980; 7: 231-236.

13. Beltrame A, et al. Imported Chkungunya infection, Italy. Emerging Infectious Diseases 2007; 13: 1264-1266.

14. Chatterjee SN, et al. Virological investigation of cases with neurological complications during the outbreak of haemorrhagic fever in Calcutta. Journal of the Indian Medical Association 1965; 45: 314-316.

15. Ravi V. Re-emergence of chikungunya virus in India. Indian Journal of Medical Microbiology 2006; 24: 83-84.

16. Obeyesekere I, Hermon Y. Arbovirus heart disease: myocarditis and cardiomyopathy following dengue and chikungunya fever - a follow-up study. American Heart Journal 1973; 85: 186-194.
17. Mirabel MM, et al. Acute myocarditis due to chikungunya virus assessed by contrast-enhanced MRI. International Journal of Cardiology 2007; 121: e7-8.

18. Schuffenecker I, et al. Genome microevolution of chikungunya viruses causing the Indian ocean outbreak. PLoS Medicine 2006; 3: e263.

19. Parola P, et al. Novel chikungunya virus variant in travelers returning from Indian Ocean islands. Emerging Infectious Diseases 2006; 12: 1493-1499.

20. Josseran L, et al. Chikungunya disease outbreak, Reunion Island. Emerging Infectious Diseases 2006, 12: 1994-1995.

21. Rezza G, et al. (CHIKV Study Group). Infection with chikungunya virus in Italy: an outbreak in a temperate region. Lancet 2007; 370: 1840-1846. 